\title{
Moderate Photoinhibition of Photosystem II Protects Photosystem I from Photodamage at Chilling Stress in Tobacco Leaves
}

\author{
Wei Huang ${ }^{1,2 * t}$, Ying-Jie Yang ${ }^{1,2 t}$, Hong Hu ${ }^{1,2}$ and Shi-Bao Zhang ${ }^{1,2 *}$ \\ ${ }^{1}$ Key Laboratory of Economic Plants and Biotechnology, Kunming Institute of Botany, Chinese Academy of Sciences, \\ Kunming, China, ${ }^{2}$ Yunnan Key Laboratory for Wild Plant Resources, Kunming, China
}

OPEN ACCESS

Edited by:

Richard Sayre,

New Mexico Consortium at Los Alamos National Labs, USA

Reviewed by:

Hazem M. Kalaji,

Warsaw University of Life Sciences,

Poland

Mikko Tikkanen

University of Turku, Finland

${ }^{*}$ Correspondence:

Wei Huang

huangwei@mail.kib.ac.cn;

Shi-Bao Zhang

sbzhang@mail.kib.ac.cn

tThese authors have contributed equally to this work.

Specialty section: This article was submitted to Plant Physiology,

a section of the journal

Frontiers in Plant Science

Received: 19 November 2015 Accepted: 02 February 2016

Published: 22 February 2016

Citation:

Huang W, Yang Y-J, Hu H and Zhang S-B (2016) Moderate Photoinhibition of Photosystem II Protects

Photosystem I from Photodamage at Chilling Stress in Tobacco Leaves.

Front. Plant Sci. 7:182.

doi: 10.3389/fpls.2016.00182
It has been indicated that photosystem I (PSI) is susceptible to chilling-light stress in tobacco leaves, but the effect of growth light intensity on chilling-induced PSI photoinhibition in tobacco is unclear. We examined the effects of chilling temperature $\left(4^{\circ} \mathrm{C}\right)$ associated with moderate light intensity $\left(300 \mu \mathrm{mol}\right.$ photons $\left.\mathrm{m}^{-2} \mathrm{~s}^{-1}\right)$ on the activities of PSI and photosystem II (PSII) in leaves from sun- and shade-grown plants of tobacco (Nicotiana tabacum cv. k326). The sun leaves had a higher activity of alternative electron flow than the shade leaves. After $4 \mathrm{~h}$ chilling treatment, the sun leaves showed significantly a higher PSI photoinhibition than the shade leaves. At chilling temperature the sun leaves showed a greater electron flow from PSII to PSI, accompanying with a lower P700 oxidation ratio. When leaves were pre-treated with lincomycin, PSII activity decreased by $42 \%$ (sun leaves) and 47\% (shade leaves) after $2 \mathrm{~h}$ exposure to the chilling-light stress, but PSI activity remained stable during the chilling-light treatment, because the electron flow from PSII to PSI was remarkably depressed. These results indicated that the stronger chilling-induced PSI photoinhibition in the sun leaves was resulted from a greater electron flow from PSII to PSI. Furthermore, moderate PSII photoinhibition depressed electron flow to PSI and then protected PSI activity against further photodamage in chilled tobacco leaves.

Keywords: chilling temperature, electron transfer, growth light intensity, photosystem I, photosystem II, photoprotection

\section{INTRODUCTION}

During the winter and spring, the combination of daytime chilling temperatures and moderate light intensity are typical climatic conditions in subtropical and temperate regions. Such conditions can cause photodamage to photosystem I (PSI) in several species, including Cucumis sativus (Sonoike and Terashima, 1994; Terashima et al., 1994; Sonoike, 1995, 1999; Kudoh and Sonoike, 2002; Zhang et al., 2011), Spinacia oleracea (Sonoike, 1995; Hwang et al., 2004), Solanum tuberosum (Havaux and Davaud, 1994), Arabidopsis thaliana (Zhang and Scheller, 2004), and Nicotiana tabacum (Barth and Krause, 1999, 2002). The electrons supplied from PSII to PSI induce the production of superoxide anion radicals that can be converted to hydrogen peroxide (Asada, 1999). This $\mathrm{H}_{2} \mathrm{O}_{2}$ reacts with reduced iron in the iron-sulfur centers to form hydroxyl radicals that immediately cause damage to those centers in the PSI complex (Sonoike et al., 1997). In previous studies on chilling stress and PSI activity, all plant 
materials were grown under low or moderate light intensities. However, in routine production, chilling-sensitive crop plants, e.g., cucumber, potato, and tobacco, are usually cultivated under field conditions that include brighter illumination. Because photosynthetic electron flow and photoprotective mechanisms such as cyclic electron flow (CEF) and no-photochemical quenching (NPQ) are affected by growth light intensity (Miyake et al., 2005), any examination of the response of PSI activity to chilling-light stress should consider the level of growth light condition. However, little is known about the influence of growth light condition on that scenario.

At normal growing temperatures (e.g., $25^{\circ} \mathrm{C}$ ) and low light, the electron flow from PSII does not exceed the capacity of PSI electron acceptors to cope with electrons, and PSI remains stable (Munekage et al., 2002; Tikkanen et al., 2010, 2014). Damage to PSI occurs only when this electron flow exceeds the capacity of those PSI acceptors (Tikkanen and Aro, 2014; Tikkanen et al., 2014). At a chilling temperature, inhibition of the Calvin Cycle can induce an increase in NADPH/NADP ${ }^{+}$, leading to the reduction in electron transport chains and the production of superoxide anion radicals (Murata et al., 2007). This subsequently causes photodamage to PSI in cucumber, spinach, and Arabidopsis thaliana (Sonoike, 2006). When the electron flow from PSII to PSI is blocked by DCMU and DBMIB, PSI photoinhibition is not observed in the chilled leaves of potato, cucumber and spinach, apparently because those electron transport chains are oxidized and production of superoxide anion radicals on the PSI acceptor side is inhibited (Havaux and Davaud, 1994; Sonoike, 1995). Therefore, based on those reports, one might conclude that electron flow from PSII is necessary for PSI photoinhibition at chilling temperatures.

Tobacco plants grown under high light have greater photosynthetic capacity and electron transport from PSII to PSI than those exposed to low light, regardless of the temperature at which measurements are made (Yamori et al., 2010a). Consequently, we speculated that, when illuminated at chilling temperature, tobacco leaves grown under high light had higher electron flow from PSII to PSI than those leaves grown under low light. Because the electron transport responding to alternative electron sinks contributes to the production of ROS in the acceptor side of PSI, the sensitivity of PSI to photoinhibition at chilling temperature is induced by alternative electron flow (Sonoike, 1995). However, it is unclear whether the difference of chilling-induced photoinhibition of PSI between tobacco sun and shade leaves is related to alternative electron flow.

Photoinhibition of PSII was regarded as an ultimate mechanism for protecting PSI activity in pgr5 mutants of Arabidopsis thaliana that lack PGR5-dependent CEF (Tikkanen et al., 2014). When PSII activity was decreased by about $40 \%$ in pgr5 plants, PSI activity was protected against further photodamage because of decreased electron flow from PSII (Tikkanen et al., 2014). In plants, CEF and NPQ are two protective mechanisms for PSII activity (Munekage et al., 2002, 2004; Takahashi et al., 2009; Brestic et al., 2014, 2015; Zivcak et al., 2014a). Activation of CEF and NPQ alleviate PSII photoinhibition at chilling temperature (Kim et al., 2001; Li et al., 2004; Huang et al., 2011). Tobacco plants grown under high light had greater capacities for CEF and NPQ when compared with plants grown under low light (Miyake et al., 2005). The extent of chilling-induced PSII photoinhibition is diminished in more brightly lit plants. The ROS production was highly correlated to PSII activity (Oukarroum et al., 2015). Thus, the higher PSII activity in those plants probably aggravates PSI photoinhibition under chilling-light stress.

Here, we investigated the response of PSI and PSII activities to $4^{\circ} \mathrm{C}$ and $300 \mu \mathrm{mol}$ photons $\mathrm{m}^{-2} \mathrm{~s}^{-1}$ in tobacco leaves grown under two different light conditions (95\% sunlight for sun leaves, $28 \%$ sunlight for shade leaves). Our aim was to examine whether the growth light intensity influences the response of PSI activity to combined chilling and light stresses. Here, PSI was more susceptible to such stress in sun leaves than shade leaves, due to higher electron flow from PSII to PSI. When PSII repair was inhibited by lincomycin, a large decrease in PSII activity limited electron flow from PSII to PSI, and thus PSI activity was not sensitive to chilling-light stress in either leaf type.

\section{MATERIALS AND METHODS}

\section{Plant Materials}

Seedlings of the ' $\mathrm{k} 326$ ' cultivar from tobacco (Nicotiana tabacum) were cultivated in plastic pots in a phytotron at Kunming Institute of Botany, Yunnan, China (elevation 1900 m, $\left.102^{\circ} 41^{\prime} \mathrm{E}, 25^{\circ} 01^{\prime} \mathrm{N}\right)$. Day/night temperatures were $24^{\circ} \mathrm{C} / 18^{\circ} \mathrm{C}$. Relative humidity was kept at $60 \%$ and the atmospheric $\mathrm{CO}_{2}$ concentration $\left(C_{\mathrm{a}}\right)$ was held at $400 \mu \mathrm{mol} \mathrm{mol}{ }^{-1}$. The phytotron used sunlight as the source of illumination, and the light intensity received by sun plants was about $95 \%$ of full sunlight (maximum intensity at noon $\approx 1990 \mu \mathrm{mol}$ photons $\left.\mathrm{m}^{-2} \mathrm{~s}^{-1}\right)$. The shade plants were grown under $28 \%$ sunlight (maximum intensity $\approx 580 \mu \mathrm{mol}$ photons $\mathrm{m}^{-2} \mathrm{~s}^{-1}$ ). During the experimental period, none of the plants experienced any water or nutrient stresses. After the plants were transplanted and cultivated for 50 days, the newly produced, mature leaves were used for photosynthetic measurements.

\section{Simultaneous Measurements of Chlorophyll Fluorescence and P700 Redox State}

A Dual-PAM-100 system (Heinz Walz, Effeltrich, Germany) was used for simultaneous measurements of chlorophyll fluorescence and the P700 redox state. In the early morning, after darkadaptation overnight, values for $F_{v} / F_{m}$ were obtained from intact mature leaves $\left(F_{v}\right.$, variable fluorescence; $F_{m}$, maximum fluorescence). Those leaves with $F_{v} / F_{m}$ values $>0.8$ were chosen for chilling treatments.

The following chlorophyll fluorescence parameters were calculated: $F_{o}{ }^{\prime}=F_{o} /\left[\left(F_{m}-F_{o}\right) / F_{m}+F_{o} / F_{m}{ }^{\prime}\right]$ (Oxborough and Baker, 1997), $\mathrm{qL}=\left(F_{m}{ }^{\prime}-F_{s}\right) /\left(F_{m}{ }^{\prime}-F_{o}{ }^{\prime}\right) \times F_{o}{ }^{\prime} / F_{s} . F_{o}$ and $F_{m}$ are the minimum and maximum fluorescence after dark-adaptation; $F_{o}{ }^{\prime}$ and $F_{m}{ }^{\prime}$ are the minimum and maximum fluorescence under light, respectively; $\mathrm{qL}$ is the coefficient of photochemical quenching based on the "lake" model (Oxborough and Baker, 
1997); $F_{S}$ is the light-adapted steady-state fluorescence; and $\mathrm{Y}(\mathrm{II})$ is the effective quantum yield of PSII under light. $F_{m}$ and $F_{m}{ }^{\prime}$ were measured upon illumination with a 300 -ms pulse of saturating light $\left(10000 \mu \mathrm{mol}\right.$ photons $\left.\mathrm{m}^{-2} \mathrm{~s}^{-1}\right)$. Because damage to PSI increases $F_{o}$ and, therefore, $F_{v} / F_{m}$ is affected by photodamage to both PSI and PSII, we used $F_{m}$ to estimate the amount of active PSII reaction centers (Tikkanen et al., 2014).

The maximum photo-oxidizable P700 was measured with a dual wavelength unit $(830 / 875 \mathrm{~nm})$ according to the method of Klughammer and Schreiber (2008). A saturation pulse (10000 $\mu \mathrm{mol}$ photons $\mathrm{m}^{-2} \mathrm{~s}^{-1}$ ) was applied for assessing P700 parameters. The $\mathrm{P} 700^{+}$signal $(P)$ varies between a minimum (P700 fully reduced) and maximum level (P700 fully oxidized). At a defined optical property, the amplitude of $P_{m}$ depends on the maximum amount of photo-oxidizable P700. As a result, the alteration in $P_{m}$ serves as an indicator of change in PSI activity (Huang et al., 2010a,b, 2013; Gao and Wang, 2012; Suorsa et al., 2012). In our present study, $P_{m}$ was measured to estimate the amount of PSI reaction centers. $P_{m}{ }^{\prime}$ was also defined in analogy to the fluorescence parameter $F_{m}{ }^{\prime} . P_{m}{ }^{\prime}$ was determined similarly to $P_{m}$, but with background actinic light instead of far-red illumination. The P700 oxidation ratio [Y(ND)] was measured as $P / P_{m}$ (Pfundel et al., 2008; Huang et al., 2011, 2012; Suorsa et al., 2012; Tikkanen et al., 2014).

\section{Simultaneous Measurements of Gas Exchange and Chlorophyll Fluorescence}

An open gas exchange system incorporating infrared $\mathrm{CO}_{2}$ and water vapor analyzers (Li-6400XT; Li-Cor Inc., Lincoln, NE, USA) was used to determine the rate of $\mathrm{CO}_{2}$ assimilation $\left(A_{\mathrm{n}}\right)$ in the phytotron. Chlorophyll fluorescence was measured simultaneously with gas exchange measurements using a fluorometer chamber (6400-40; Li-Cor Inc.). The fluorescence parameters $F_{s}$ and $F_{m}{ }^{\prime}$ were determined as previously described (Baker and Rosenqvist, 2004), with $F_{s}$ representing the steady fluorescence and $F_{m}^{\prime}$ the maximum fluorescence after lightadaptation. The effective quantum yield of PSII was calculated as $\Phi_{\text {PSII }}=\left(F_{m}^{\prime}-F_{s}\right) / F_{m}^{\prime}$ (Genty et al., 1989). During the measurement period, the relative air humidity was $60 \%$ and the air temperature was $24^{\circ} \mathrm{C}$. To generate a light response curve, the leaves of both sun and shade plants were exposed to high light (i.e., $1200 \mu \mathrm{mol}$ photons $\mathrm{m}^{-2} \mathrm{~s}^{-1}$ ) for $20 \mathrm{~min}$ to obtain a steady state. Afterward, photosynthetic parameters were evaluated every $2 \mathrm{~min}$ at a controlled $C_{\mathrm{a}}$ of $400 \mu \mathrm{mol}$ $\mathrm{mol}^{-1}$ and photosynthetic photon flux densities (PPFDs) of $2000,1600,1200,800,500,300,200,100,50,20$, or $0 \mu \mathrm{mol}$ photons $\mathrm{m}^{-2} \mathrm{~s}^{-1}$. The PSII electron transport rate $\left(J_{F}\right)$ based on chlorophyll fluorescence measurement was calculated as $J_{F}=0.85 \times 0.5 \times$ PPFD $\times \Phi_{\text {PSII }}$ (Miyake et al., 2005; Zhang et al., 2013; Huang et al., 2014, 2015). The rate of electron transport consumed by carboxylation plus oxygenation of RuBP $\left(J_{G}\right)$ was calculated as $J_{G}=4\left(A_{n}+R_{d}\right)\left(C_{i}+2 \Gamma^{*}\right) /\left(C_{i}-\Gamma^{*}\right)$ (Harley et al., 1992; Zivcak et al., 2013), where $A_{n}$ represents measured $\mathrm{CO}_{2}$ assimilation rate, $R_{d}$ represents the mitochondrial respiration measured after 5 min dark adaptation, $C_{i}$ represents the intercellular $\mathrm{CO}_{2}$ concentration, and $\Gamma^{*}$ represents the $\mathrm{CO}_{2}$ compensation point measured in the absence of respiration. The value of $\Gamma^{*}$ was calculated to be 32.2 at $25^{\circ} \mathrm{C}$ according to Long and Bernacchi (2003).

\section{Photoinhibitory Treatment at $4^{\circ} \mathrm{C}$}

To examine the effect of growth light condition on chillinginduced PSI photoinhibition, detached sun and shade leaves incubated with water overnight in darkness were transferred to $4^{\circ} \mathrm{C}$ and $300 \mu \mathrm{mol}$ photons $\mathrm{m}^{-2} \mathrm{~s}^{-1}$. To examine the effect of PSII photoinhibition on chilling-induced PSI photoinhibition, detached sun and shade leaves incubated in the presence of lincomycin (Lin, $1 \mathrm{mM}$ ) overnight in darkness were transferred to $4^{\circ} \mathrm{C}$ and $300 \mu \mathrm{mol}$ photons $\mathrm{m}^{-2} \mathrm{~s}^{-1}$. Before chilling-light treatment, $\mathrm{qL}$ and $\mathrm{Y}(\mathrm{ND})$ were measured at $25^{\circ} \mathrm{C}$ and $297 \mu \mathrm{mol}$ photons $\mathrm{m}^{-2} \mathrm{~s}^{-1}$. After chilling-light treatment for 2, 4, and $6 \mathrm{~h}$, $\mathrm{qL}$ and $\mathrm{Y}(\mathrm{ND})$ were measured immediately at $4^{\circ} \mathrm{C}$ and $297 \mu \mathrm{mol}$ photons $\mathrm{m}^{-2} \mathrm{~s}^{-1}$. Subsequently, $P_{m}$ and $F_{m}$ were measured after 30 min dark adaptation.

\section{Statistical Analysis}

All results were displayed as mean values of six independent experiments. Data were subjected to an Independent-Samples $T$-test with SPSS 16.0 statistical software. Independent-Samples $T$-test was used at $\alpha=0.05$ significance level to determine whether significant differences existed between different treatments.

\section{RESULTS}

\section{Alternative Electron Flow in the Sun and Shade Leaves}

To estimate the linear electron flow that is not used for RuBP carboxylation and photorespiration, PSII electron flow calculated from chlorophyll fluorescence measurements $\left(J_{\mathrm{F}}\right)$ and electron transport calculated from gas exchange $\left(J_{\mathrm{G}}\right)$ was compared in the sun and shade leaves (Figures 1A,B). The difference between $J_{\mathrm{F}}$ and $J_{\mathrm{G}}$ represents the electron flow utilized by alternative electron sinks. Light response curves indicated that under high light the sun leaves had significantly higher values of $J_{\mathrm{F}}$ and $J_{\mathrm{G}}$ at $25^{\circ} \mathrm{C}$ (Figures 1A,B), due to higher rate of $\mathrm{CO}_{2}$ assimilation and photorespiration (Huang et al., 2014). Furthermore, the value of $J_{F}-J_{G}$ largely differed between the sun and shade leaves (Figure 1C), indicating the sun leaves had significantly a higher capacity of alternative electron flow than the shade leaves.

\section{Photoinhibition of PSI and PSII}

To examine the effect of growth light condition on chillinginduced PSI photoinhibition, detached sun and shade leaves incubated with water overnight in darkness were transferred to $4^{\circ} \mathrm{C}$ and $300 \mu \mathrm{mol}$ photons $\mathrm{m}^{-2} \mathrm{~s}^{-1}$. By contrast, exposure for $4 \mathrm{~h}$ was associated with declines in $P_{m}$ of $28 \%$ and $14 \%$ for sun and shade leaves, respectively (Figure 2A). This indicated that PSI activity was more sensitive to chilling-light stress in the sun leaves. However, prolonging the chilling period did not enhance PSI photodamage in either sun or shade leaves (Figure 2A). 


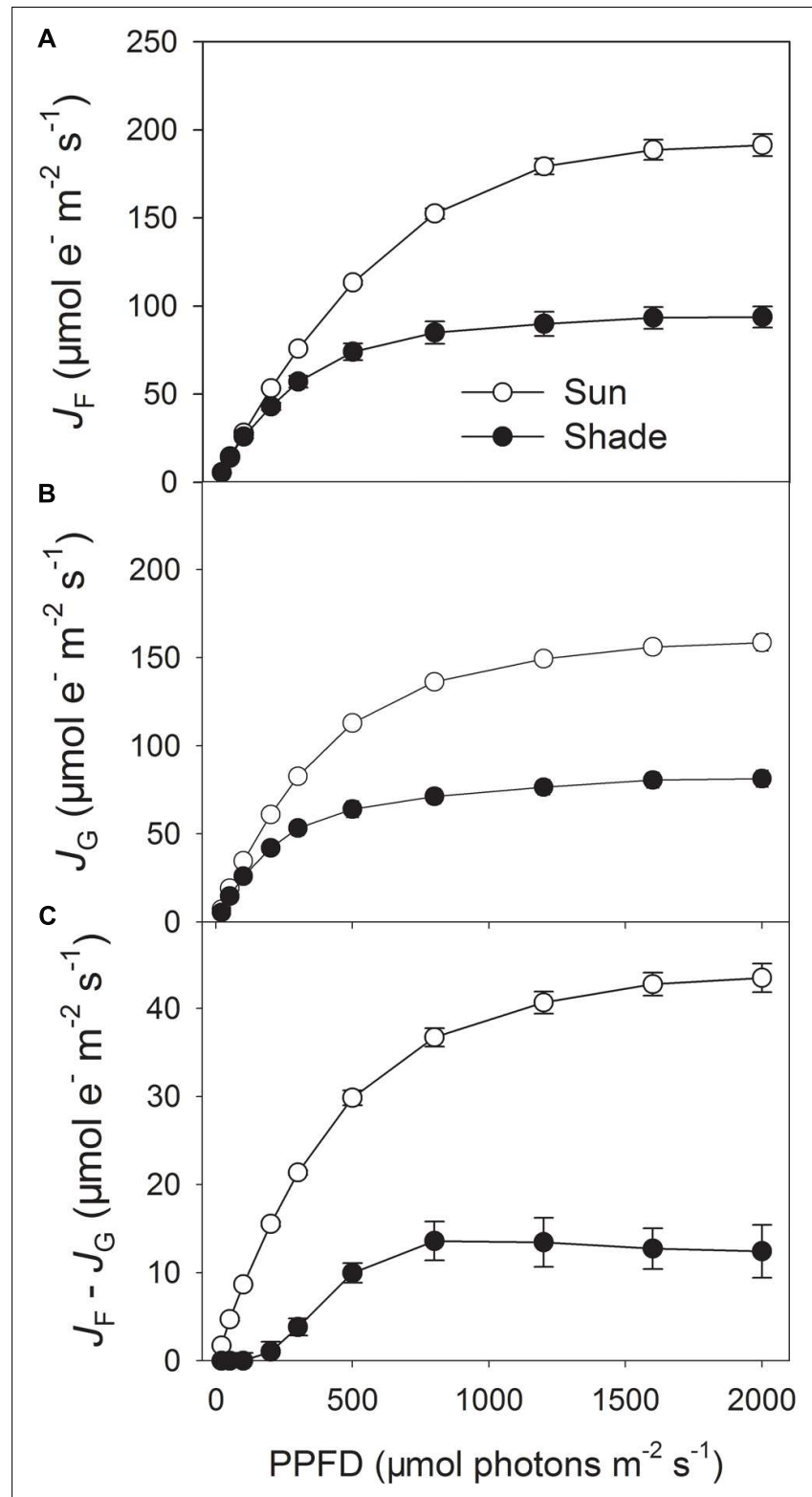

FIGURE 1 | Light response changes in photosynthetic electron flow in the sun and shade leaves of tobacco. (A) PSIl electron transport rate $\left(J_{F}\right)$ calculated based on measurements of PSIl quantum yields, assuming the equal distribution of absorbed light between PSI and PSII. (B) Rate of electron transport consumed by carboxylation plus oxygenation of RuBP $\left(\mathrm{J}_{\mathrm{G}}\right)$, calculated by the data from gas exchange measurements. (C) The difference between $J_{F}$ and $J_{G}, J_{F}-J_{G}$ represents a common way to estimate alternative electron flow besides the Calvin cycle and photorespiration. Values are means \pm SE $(n=6)$.

Under such stress, shade leaves showed higher PSII photoinhibition when compared with the sun leaves. For example, after 2,4 , and $6 \mathrm{~h}$ of treatment, $F_{m}$ values decreased by 22,34 , and $40 \%$ in the sun leaves, respectively, versus declines of 26, 39, and $48 \%$ in the shade leaves (Figure 2B). The extent of PSII photoinhibition differed slightly between the two types. In the initial $4 \mathrm{~h}$ chilling treatment, the reduction in PSII activity was accompanied by a decrease in PSI activity for both type leaves (Figures 2A,B). Between hours 4 and 6, PSII activity continued to drop whereas that of PSI was unaffected (Figures 2A,B). These results suggested that the chilling-induced decrease in PSI activity was dependent on a high PSII activity, and that PSI might have been protected from further photodamage while PSII activity declined by approximately $40 \%$.

To further understand the effect of chilling-induced PSII photoinhibition on PSI activity, detached leaves incubated with lincomycin overnight in darkness were exposed to the above chilling-light stress. Although neither the sun nor the shade leaves showed a significant reduction in $P_{m}$ values (Figure 2C), the $F_{m}$ for both leaf types was largely decreased. For example, after exposure to the combined stress for 2, 4, and $6 \mathrm{~h}, F_{m}$ dropped by 42,44 , and $46 \%$, respectively, in the sun leaves, and by 47,49 , and $54 \%$, respectively, in the shade leaves (Figure 2D). Therefore, in the presence of lincomycin, PSII photoinhibition was aggravated and then photodamage to PSI was prevented. This strongly suggested that chilling-induced PSI photoinhibition was dependent on PSII activity.

\section{Relative $\mathbf{Q}_{A}$ Reduction and PSI Redox State}

To understand the effect of chilling-induced PSII photoinhibition on relative $\mathrm{Q}_{\mathrm{A}}$ reduction and PSI redox state, the changes in $\mathrm{qL}$ and $\mathrm{Y}(\mathrm{ND})$ during chilling treatment were measured. During chilling-light treatment, $\mathrm{qL}$ decreased gradually in both the sun and shade leaves (Figure 3A). The sun leaves showed significantly higher $\mathrm{qL}$ values than the shade leaves during chilling-light treatment (Figure 3A). This result implied that at chilling temperature the sun leaves displayed higher electron flow from PSII to PSI. With increasing time of chilling-treatment, $\mathrm{Y}(\mathrm{ND})$ gradient increased in both type leaves. Furthermore, the $\mathrm{Y}(\mathrm{ND})$ values were lower in the sun leaves compared with the shade leaves (Figure 3B). Interestingly, the value of $\mathrm{Y}(\mathrm{ND})$ was lower than 0.2 in the sun leaves after $2 \mathrm{~h}$ chilling treatment, implying the over-reduction of PSI acceptor side. In the presence of lincomycin, qL largely decreased after initial $2 \mathrm{~h}$ exposure to chilling-light stress in both the sun and shade leaves (Figure 3C). Meanwhile, Y(ND) largely increased in them (Figure 3D). These results indicated that, when down-regulation of PSII activity was induced by mild lincomycin treatment, the electron flow from PSII to PSI was limited, resulting in the decrease in $\mathrm{qL}$ and the increase in $\mathrm{Y}(\mathrm{ND})$.

\section{DISCUSSION}

\section{PSI Activity in Tobacco is More Sensitive to Chilling-Light Stress in Sun Leaves}

Previous studies indicated that PSI activity was sensitive to chilling-light stress in tobacco leaves grown under low light (Barth and Krause, 1999, 2002). However, the effect of chillinglight stress on PSI activity is unclear for tobacco leaves grown under high light. Our results strongly indicated that chillinginduced PSI photoinhibition was significantly stronger in the sun 

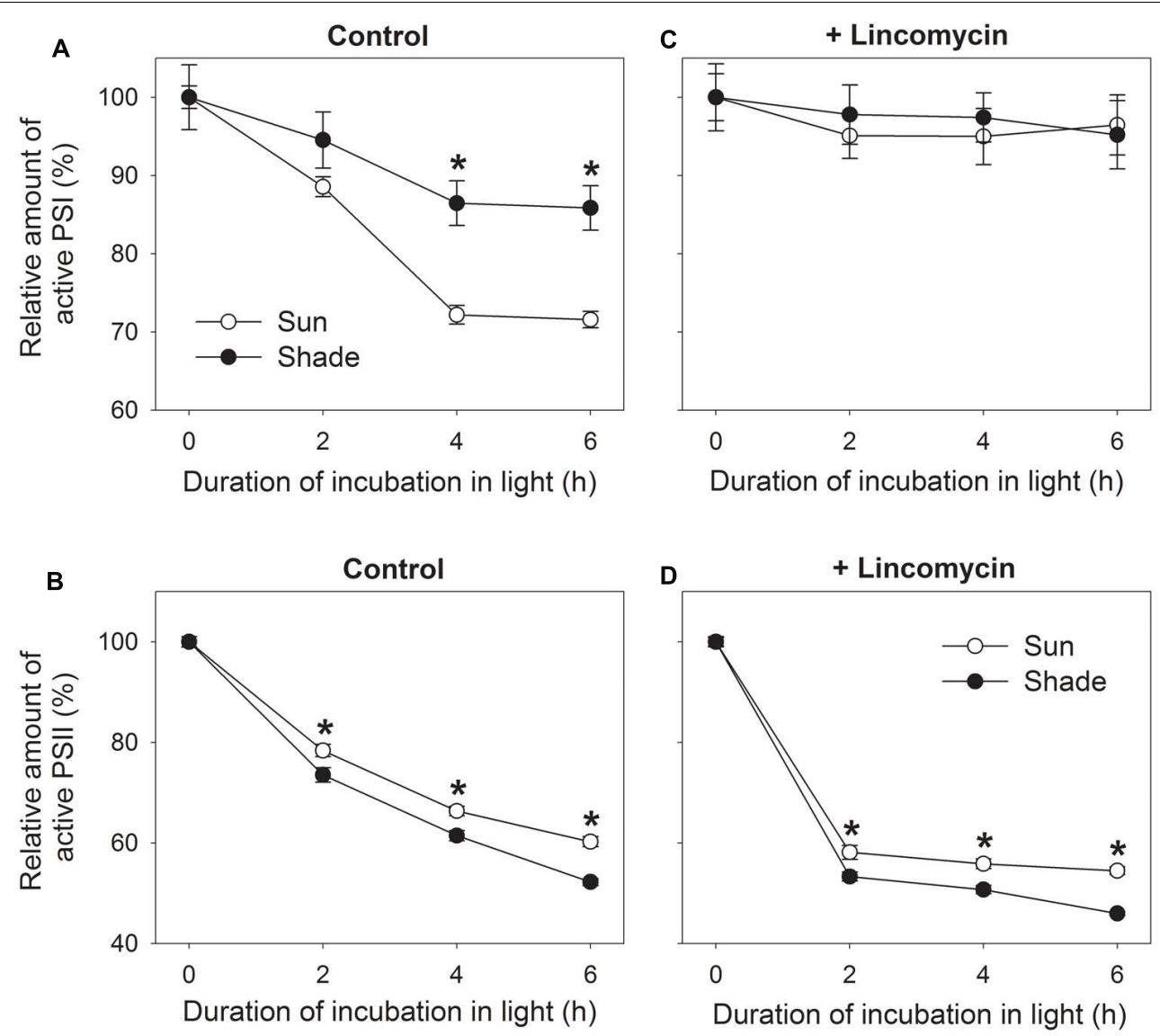

FIGURE 2 | Relationship between photoinhibition of PSII (B,D) and PSI (A,C) in sun and shade tobacco leaves. Detached leaves incubated in the presence or absence of lincomycin (1 mM) overnight in darkness were exposed to $4^{\circ} \mathrm{C}$ and $300 \mu \mathrm{mol}$ photons $\mathrm{m}^{-2} \mathrm{~s}^{-1}$ for 2,4, or $6 \mathrm{~h}$. $F_{m}$ was measured after dark adaptation to estimate the amount of active PSIl centers. $P_{m}$ was measured after dark adaptation to estimate the amount of active PSI centers. All values are expressed relative to the controls before chilling-light treatment, and shown as means $\pm \operatorname{SE}(n=6)$. Asterisks indicate significant differences between the sun and shade leaves.

leaves than the shade leaves (Figure 2A). This suggested that the extent of chilling-induced PSI photoinhibition was influenced by growth light intensity. The effect of growth irradiance on chilling-induced PSI photoinhibition was previously examined in common bean (Sonoike et al., 1995). Common bean leaves grown in $6.5 \%$ of full sunlight displayed stronger chilling-induced PSI photoinhibition than those grown in full sunlight (Sonoike et al., 1995). On the contrary, our results indicated that PSI was more sensitive to chilling-light stress in sun leaves than shade leaves in tobacco. Thus, we assumed that the effect of growth light condition on chilling-induced PSI photoinhibition strongly depended on plant species.

It has been indicated that, when exposed to chillinglight stress, the inhibition of Calvin cycle decreases the $\mathrm{NADP}^{+} / \mathrm{NADPH}$ ratio and leads to the generation of superoxide anion radicals (Murata et al., 2007), which can be converted into $\mathrm{H}_{2} \mathrm{O}_{2}$ (Asada, 1999). In the presence of reduced metal ions, this $\mathrm{H}_{2} \mathrm{O}_{2}$ is converted to the hydroxyl radical, which is highly reactive and destroys the iron-sulfur centers on the acceptor side of PSI (Sonoike, 1995, 2006, 2011). Excess electron flow from PSII to PSI can lead to reduction of PSI acceptors and production of superoxide anion radicals (Oukarroum et al., 2015), as a result, PSI only gets photodamaged when electron transfer to PSI is in excess of the capacity of PSI electron acceptors (Tikkanen and Aro, 2014; Tikkanen et al., 2014). Our results indicated that the sun leaves of tobacco had a higher capacity of alternative electron flow than the shade leaves (Figure 1C). The alternative electron flow is mainly caused by photoreduction of $\mathrm{O}_{2}$, which generates ROS at the acceptor side of PSI. During chilling-light treatment, the sun leaves had higher qL values than the shade leaves (Figure 3A). More electrons being transferred to PSI in the sun leaves not only led to stronger production of superoxide anion radicals in PSI acceptor side, but also increased the P700 reduction ratio. Taking together, the higher PSI photoinhibition in the sun leaves was significantly related to the higher alternative electron flow at chilling-light stress.

In plants, CEF can protect PSI against photodamage under high light by preventing over-reduction on the acceptor side in PSI (Munekage et al., 2002, 2004; Suorsa et al., 2012; Tikkanen et al., 2014; Zivcak et al., 2014a; Brestic et al., 2015). At a chilling temperature, CEF alleviates PSI photoinhibition in 

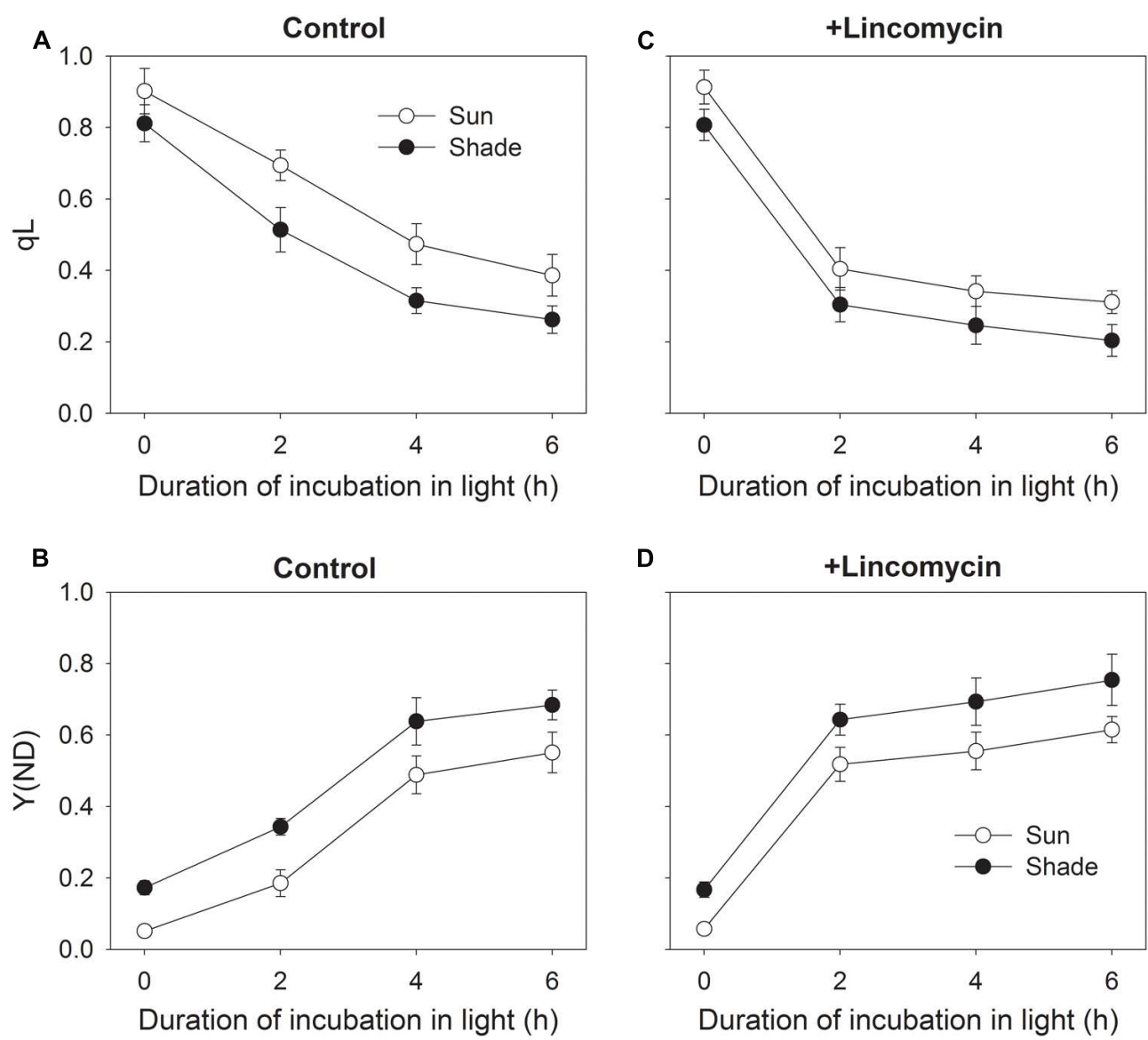

D

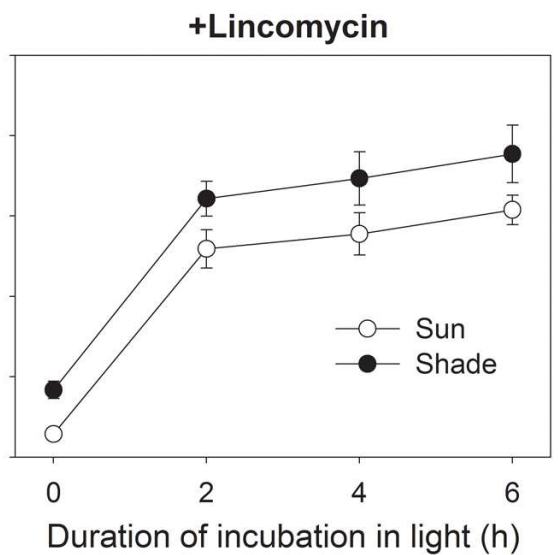

FIGURE 3 | Changes in relative $Q_{A}$ reduction (qL) (A,C) and PSI oxidation ratio [Y(ND)] (B,D) during exposure to $4^{\circ} \mathrm{C}_{\text {and }} 300 \mu \mathrm{mol}^{\mathrm{photons}} \mathrm{m}^{-2} \mathrm{~s}^{-1}$. After measuring $\mathrm{qL}$ and $\mathrm{Y}(\mathrm{ND})$ at $24^{\circ} \mathrm{C}$ and $297 \mu \mathrm{mol}$ photons $\mathrm{s}^{-2} \mathrm{~s}^{-1}$, leaves were incubated in the presence or absence of lincomycin (1 mM) overnight in darkness and subsequently exposed to $4^{\circ} \mathrm{C}$ and $300 \mu \mathrm{mol}$ photons $\mathrm{m}^{-2} \mathrm{~s}^{-1}$ for 2,4 , or $6 \mathrm{~h}$. During chilling-light treatment, $\mathrm{qL}$ and $\mathrm{Y}(\mathrm{ND})$ were measured at $4^{\circ} \mathrm{C}$ and $297 \mu \mathrm{mol}$ photons $\mathrm{m}^{-2} \mathrm{~s}^{-1}$. Values are means $\pm \operatorname{SE}(n=6)$.

cucumber (Kim et al., 2001; Bukhov et al., 2004) and tropical tree species (Huang et al., 2011). The capacity for CEF can also be affected by the growth light intensity to which tobacco plants are exposed, and the tobacco sun leaves have higher CEF capacity than the shade leaves (Miyake et al., 2005; Huang et al., 2015). If CEF in tobacco did in fact have a major role in protecting PSI activity against photodamage at chillinglight stress, then we would expect the sun leaves to have less PSI photoinhibition. On the contrary, the sun leaves showed significantly stronger PSI photoinhibition, indicating that CEF provided only minimal photoprotection for PSI in tobacco leaves at chilling-light stress. The slight difference in chillinginduced PSII photoinhibition between the sun and shade leaves further indicated that CEF was hardly activated at chilling temperature in tobacco leaves. Barth and Krause (2002) indicated that $\mathrm{NAD}(\mathrm{P}) \mathrm{H}$ dehydrogenase $(\mathrm{NDH})$-mediated $\mathrm{CEF}$ did not protect PSI against short chilling-light stress. Furthermore, $\mathrm{NDH}$-dependent CEF was less important under chilling-stressed condition (Wang et al., 2006). Thus, CEF hardly prevented PSI photoinhibition in tobacco leaves illuminated at chilling temperature.

\section{Moderate PSII Photoinhibition Prevents PSI Photoinhibition Under Chilling-Light Stress}

Our results clearly demonstrate that chilling-induced photoinhibition of PSI in tobacco leaves is dependent upon PSII activity. In the absence of lincomycin, PSI activity decreased during the first $4 \mathrm{~h}$ of chilling treatment (Figure 2A). However, longer exposure to chilling-light stress did not aggravate PSI photoinhibition in either sun or shade leaves (Figure 2A). After $4 \mathrm{~h}$ of treatment, PSII activity decreased by 34 and $39 \%$ in sun and shade leaves, respectively. Meanwhile, qL largely decreased in both the sun and shade leaves (Figure 3A). This large decrease in $\mathrm{qL}$ led to a decline in electron transfer from PSII to PSI, and then increase P700 oxidation ratio. Consequently, there was no significant decrease in PSI activity between 4 and $6 \mathrm{~h}$ of chilling-light treatment in both type leaves. In the presence of lincomycin, PSII activity decreased by 42 and $47 \%$ after $2 \mathrm{~h}$ in stressed sun and shade leaves, respectively. This large decrease in PSII activity led to a depression of linear electron flow, as indicated by the decrease in $\mathrm{qL}$ and increase in $\mathrm{Y}(\mathrm{ND})$ 
(Figures 3C,D). Moreover, PSI activity was maintained stable in either leaf type after $6 \mathrm{~h}$ chilling-light treatment in the presence of lincomycin. Therefore, when PSII activity decreased by about $40 \%$, PSI activity was protected from further chilling-induced photodamage.

Photosystem I becomes irreversibly photodamaged if the electrons supplied from PSII to PSI exceed the capacity of PSI electron acceptors. At normal growing temperatures and low light, the electrons transported from PSII to PSI can be efficiently quenched by the Calvin cycle and photorespiratory pathway. However, when plants are subjected to a chilling temperature, the electrons transported to PSI cannot be efficiently quenched through the Calvin cycle and photorespiratory pathway, which then leads to a reduction in photosynthetic electron chains and the production of superoxide anion radicals. In other chillingsensitive species, such as cucumber and Arabidopsis thaliana, chilling-light stress induces a slight decrease in PSII activity but a large decrease in PSI activity (Sonoike, 1995; Zhang and Scheller, 2004), indicating that the slight decrease in PSII activity has little influence on electron flow from PSII to PSI. The CEFdeficient pgr5 plants showed large PSI photoinhibition upon shift to high light. However, when the PSII repair was inhibited by lincomycin in pgr5 plants, moderate PSII photoinhibition led to a depression of linear electron flow and then protected PSI against further photodamage in pgr5 plants (Tikkanen et al., 2014). In the samples pre-treated with lincomycin, the chillinglight stress did not cause significant PSI photoinhibition in either sun or shade leaves. Meanwhile, the Lin-treated samples had significantly lower $\mathrm{qL}$ and higher $\mathrm{Y}(\mathrm{ND})$ than the $\mathrm{H}_{2} \mathrm{O}$-treated samples in the initial $2 \mathrm{~h}$ exposure to the chilling-light stress. This depression of electron flow from PSII to PSI following a decline in PSII activity increased the level of P700 oxidation and diminished the production of superoxide anion radicals (Tikkanen et al., 2014; Oukarroum et al., 2015). Taken together, our data support the proposal that moderate down-regulation of PSII has the potential role in protecting PSI activity against further photodamage at chilling-light stress.

\section{CONCLUSION}

Plants regulate photosynthetic machinery to acclimate different growth conditions including changes in irradiance (Yamori et al., 2010a; Huang et al., 2014; Zivcak et al., 2014b), nutrients (Hikosaka, 1996; Kalaji et al., 2014), temperature (Yamori et al., 2010b, 2011), and water availability (Lehtimaki et al., 2010).

\section{REFERENCES}

Asada, K. (1999). The water-water cycle in chloroplasts: scavenging of active oxygens and dissipation of excess photons. Annu. Rev. Plant Biol. 50, 601-639. doi: 10.1146/annurev.arplant.50.1.601

Baker, N. R., and Rosenqvist, E. (2004). Applications of chlorophyll fluorescence can improve crop production strategies: an examination of future possibilities. J. Exp. Bot. 55, 1607-1621. doi: 10.1093/jxb/erh196

Barth, C., and Krause, G. H. (1999). Inhibition of photosystem I and II in chillingsensitive and chilling-tolerant plants under light and low-temperature stress. Z. Naturforsch. 54c, 645-657.
The shade leaves have a high capacity of light reactions as compared to the capacity of the sink. Because of this, chillinglight treatment is able to induce higher lumenal protonation in the shade leaves than the sun leaves, resulting in slowdown of cytochrome $b_{6} / f$ in the shade leaves (Tikkanen and Aro, 2014). The sun leaves had higher alternative electron flow than the shade leaves. Furthermore, the higher $\mathrm{qL}$ values at chilling-light stress indicated higher alternative electron sinks such as photoreduction of $\mathrm{O}_{2}$ that produces $\mathrm{O}_{2}{ }^{-}$, causing stronger PSI photoinhibition in the sun leaves. Furthermore, the lower connectivity between PSII units in the shade leaves limited electron transport between PSII and PSI (Zivcak et al., 2014a), which alleviated PSI photoinhibition at chilling-light stress. When PSII photoinhibition was aggravated by the addition of lincomycin, PSI activity was insusceptible to chilling-light stress in both sun and shade leaf types, as a result of lower $\mathrm{qL}$ values and higher $\mathrm{Y}(\mathrm{ND})$ values. Therefore, moderate PSII photoinhibition depressed the electron flow from PSII to PSI and thus alleviated PSI photoinhibition. Our results strongly supported the hypothesis that photoinhibition of PSI occurs only when electron flow to PSI exceeds the capacity of PSI electron acceptors as proposed by recent studies (Suorsa et al., 2012; Tikkanen and Aro, 2014; Tikkanen et al., 2014). Because of the importance of PSI in photosynthetic regulation, when tobacco sun leaves are exposed to long-term chilling-light stress, a strong irreversible photodamage of PSI can lead to severe photoinhibition of PSII and finally to the death of the plant. During the short-term chilling treatment, PSII photoinhibition can be regarded as an important mechanism protecting PSI against further photoinhibition in tobacco.

\section{AUTHOR CONTRIBUTIONS}

$\mathrm{WH}$ and S-BZ conceived and designed research. WH and Y-JY conducted experiments. WH contributed new reagents or analytical tools. WH, Y-JY, and S-BZ analyzed data. WH, Y-JY, $\mathrm{S}-\mathrm{BZ}$, and $\mathrm{HH}$ wrote the manuscript.

\section{ACKNOWLEDGMENTS}

This work was supported by the National Natural Science Foundation of China (Grant 31300332) and by the China Postdoctoral Science Foundation (Grants 2013M531994 and $2014 \mathrm{~T} 70892$ to $\mathrm{WH})$.

Barth, C., and Krause, G. H. (2002). Study of tobacco transformants to assess the role of chloroplastic $\mathrm{NAD}(\mathrm{P}) \mathrm{H}$ dehydrogenase in photoprotection of photosystems I and II. Planta 216, 273-279. doi: 10.1007/s00425-002-0843-0

Brestic, M., Zivcak, M., Kunderlikova, K., Sytar, O., Shao, H.-B., Kalaji, H. M., et al. (2015). Low PSI content limits the photoprotection of PSI and PSII in early growth stages of chlorophyll b-deficient wheat mutant lines. Photosynth. Res. 125, 151-166. doi: 10.1007/s11120-015-0093-1

Brestic, M., Zivcak, M., Olsovska, K., Shao, H.-B., Kalaji, H. M., and Allakhverdiev, S. I. (2014). Reduced glutamine synthetase activity plays a role in control of photosynthetic responses to high light in barley leaves. Plant Physiol. Biochem. 81, 74-83. doi: 10.1016/j.plaphy.2014.01.002 
Bukhov, N. G., Govindachary, S., Rajagopal, S., Joly, D., and Carpentier, R. (2004). Enhanced rates of P700+ dark-reduction in leaves of Cucumis sativus L. photoinhibited at chilling temperature. Planta 218, 852-861. doi: 10.1007/s00425-003-1165-6

Gao, S., and Wang, G. C. (2012). The enhancement of cyclic electron flow around photosystem I improves the recovery of severely desiccated Porphyra yezoensis (Bangiales, Rhodophyta). J. Exp. Bot. 63, 4349-4358. doi: 10.1093/jxb/ ers082

Genty, B., Briantais, J. M., and Baker, N. R. (1989). The relationship between the quantum yield of photosynthetic electron transport and quenching of chlorophyll fluorescence. Biochim. Biophys. Acta 990, 87-92. doi: 10.1016/S0304-4165(89)80016-9

Harley, P. C., Loreto, F., Marco, G. D., and Sharkey, T. D. (1992). Theoretical considerations when estimating the mesophyll conductance to $\mathrm{CO}_{2}$ flux by analysis of the response of photosynthesis to $\mathrm{CO}_{2}$. Plant Physiol. 98, 1429-1436.

Havaux, M., and Davaud, A. (1994). Photoinhibition of photosynthesis in chilled potato leaves is not correlated with a loss of photosystem II activity preferential inactivation of photosystem I. Photosynth. Res. 40, 75-92. doi: 10.1007/BF00019047

Hikosaka, K. (1996). Effects of leaf age, nitrogen nutrition and photon flux density on the organization of the photosynthetic apparatus in leaves of a vine (Ipomoea tricolor Cav.) grown horizontally to avoid mutual shading of leaves. Planta 198, 144-150. doi: 10.1007/BF00197597

Huang, W., Fu, P. L., Jiang, Y. J., Zhang, J. L., Zhang, S. B., Hu, H., et al. (2013). Differences in the responses of photosystem I and photosystem II of three tree species Cleistanthus sumatranus, Celtis philippensis and Pistacia weinmannifolia submitted to a prolonged drought in a tropical limestone forest. Tree Physiol. 33, 211-220. doi: 10.1093/treephys/tps132

Huang, W., Yang, S. J., Zhang, S. B., Zhang, J. L., and Cao, K. F. (2012). Cyclic electron flow plays an important role in photoprotection for the resurrection plant Paraboea rufescens under drought stress. Planta 235, 819-828. doi: 10.1007/s00425-011-1544-3

Huang, W., Yang, Y. J., Hu, H., and Zhang, S. B. (2015). Different roles of cyclic electron flow around photosystem I under sub-saturating and saturating light intensities in tobacco leaves. Front. Plant Sci. 6:923. doi: $10.3389 /$ fpls.2015.00923

Huang, W., Zhang, S. B., and Cao, K. F. (2010a). The different effects of chilling stress under moderate illumination on photosystem II compared with photosystem I and subsequent recovery in tropical tree species. Photosynth. Res. 103, 175-182. doi: 10.1007/s11120-010-9539-7

Huang, W., Zhang, S. B., and Cao, K. F. (2010b). Stimulation of cyclic electron flow during recovery after chilling-induced photoinhibition of PSII. Plant Cell Physiol. 51, 1922-1928. doi: 10.1093/pcp/pcq144

Huang, W., Zhang, S. B., and Cao, K. F. (2011). Cyclic electron flow plays an important role in photoprotection of tropical trees illuminated at temporal chilling temperature. Plant Cell Physiol. 52, 297-305. doi: 10.1093/pcp/p cq166

Huang, W., Zhang, S. B., and Hu, H. (2014). Sun leaves up-regulate the photorespiratory pathway to maintain a high rate of $\mathrm{CO}_{2}$ assimilation in tobacco. Front. Plant Sci. 5:688. doi: 10.3389/fpls.2014.00688

Hwang, H. J., Kim, J. H., Eu, Y. J., Moon, B. Y., Cho, S. H., and Lee, C. H. (2004). Photoinhibition of photosystem I is accelerated by dimethyldithiocarbamate, an inhibitor of superoxide dismutase, during light-chilling of spinach leaves J. Photochem. Photobiol. B Biol. 73, 79-85. doi: 10.1016/j.jphotobiol.2003. 09.008

Kalaji, H. M., Oukarroum, A., Alexandrov, V., Kouzmanova, M., Brestic, M., Zivcak, M., et al. (2014). Identification of nutrient deficiency in maize and tomato plants by in vivo chlorophyll a fluorescence measurements. Plant Physiol. Biochem. 81, 16-25. doi: 10.1016/j.plaphy.2014.03.029

Kim, S. J., Lee, C. H., Hope, A. B., and Chow, W. S. (2001). Inhibition of photosystems I and II and enhanced back flow of photosystem I electrons in cucumber leaf discs chilled in the light. Plant Cell Physiol. 42, 842-848. doi: $10.1093 /$ pcp/pce109

Klughammer, C., and Schreiber, U. (2008). Saturation pulse method for assessment of energy conversion in PSI. PAM Appl. Notes 1, 11-14.

Kudoh, H., and Sonoike, K. (2002). Irreversible damage to photosystem I by chilling in the light: cause of the degradation of chlorophyll after returning to normal growth temperature. Planta 215, 541-548.
Lehtimaki, N., Lintala, M., Allahverdiyeva, Y., Aro, E. M., and Mulo, P. (2010). Drought stress-induced upregulation of components involved in ferredoxindependent cyclic electron transfer. J. Plant Physiol. 167, 1018-1022. doi: 10.1016/j.jplph.2010.02.006

Li, X. G., Duan, W., Meng, Q. W., Zou, Q., and Zhao, S. J. (2004). The function of chloroplastic $\mathrm{NAD}(\mathrm{P}) \mathrm{H}$ dehydrogenase in tobacco during chilling stress under low irradiance. Plant Cell Physiol. 45, 103-108. doi: 10.1093/pcp/pch011

Long, S. P., and Bernacchi, C. J. (2003). Gas exchange measurements, what can they tell us about the underlying limitations to photosynthesis? Procedures and sources of error. J. Exp. Bot. 54, 2393-2401. doi: 10.1093/jxb/erg262

Miyake, C., Horiguchi, S., Makino, A., Shinzaki, Y., Yamamoto, H., and Tomizawa, K. (2005). Effects of light intensity on cyclic electron flow around PSI and its relationship to non-photochemical quenching of chl fluorescence in tobacco leaves. Plant Cell Physiol. 46, 1819-1830. doi: 10.1093/pcp/pci197

Munekage, Y., Hashimoto, M., Miyake, C., Tomizawa, K. I., Endo, T., Tasaka, M., et al. (2004). Cyclic electron flow around photosystem I is essential for photosynthesis. Nature 429, 579-582. doi: 10.1038/nature02598

Munekage, Y., Hojo, M., Meurer, J., Endo, T., Tasaka, M., and Shikanai, T. (2002). PGR5 is involved in cyclic electron flow around photosystem I and is essential for photoprotection in Arabidopsis. Cell 110, 361-371. doi: 10.1016/S00928674(02)00867-X

Murata, N., Takahashi, S., Nishiyama, Y., and Allakhverdiev, S. I. (2007). Photoinhibition of photosystem II under environmental stress. Biochim. Biophys. Acta 1767, 414-421. doi: 10.1016/j.bbabio.2006.11.019

Oukarroum, A., Bussotti, F., Goltsev, V., and Kalaji, H. M. (2015). Correlation between reactive oxygen species production and photochemistry of photosystems I and II in Lemna gibba L. plants under salt stress. Environ. Exp. Bot. 109, 80-88. doi: 10.1016/j.envexpbot.2014.08.005

Oxborough, K., and Baker, N. R. (1997). Resolving chlorophyll a fluorescence images of photosynthetic efficiency into photochemical and nonphotochemical components - calculation of $\mathrm{qP}$ and $\mathrm{Fv} / \mathrm{Fm}$ without measuring Fo. Photosynth. Res. 54, 135-142. doi: 10.1023/A:10059368 23310

Pfundel, E., Klughammer, C., and Schreiber, U. (2008). Monitoring the effects of reduced PS II antenna size on quantum yields of photosystems I and II using the Dual-PAM-100 measuring system. PAM Appl. Notes 1, 21-24.

Sonoike, K. (1995). Selective photoinhibition of photosystem I in isolated thylakoid membranes from cucumber and spinach. Plant Cell Physiol. 36, 825-830.

Sonoike, K. (1999). The different roles of chilling temperatures in the photoinhibition of photosystem I and photosystem II. J. Photochem. Photobiol. B Biol. 48, 136-141. doi: 10.1016/S1011-1344(99)00030-5

Sonoike, K. (2006). "Photoinhibition and protection of photosystem I," in Photosystem I: The Light-Driven Plastocyanin: Ferredoxin Oxidoreductase, ed. J. H. Golbeck (Dordrecht: Springer), 657-668.

Sonoike, K. (2011). Photoinhibition of photosystem I. Physiol. Plant. 142, 56-64. doi: 10.1111/j.1399-3054.2010.01437.x

Sonoike, K., Ishibashi, M., and Watanabe, A. (1995). "Chilling sensitive steps in leaves of Phaseolus vulgaris L. Examination of the effects of growth irradiances on PSI photoinhibition," in Photosynthesis: From Light to Biosphere, ed. P. Mathis (Dordrecht: Kluwer Academic Publishers), 853-856.

Sonoike, K., Kamo, M., Hihara, Y., Hiyama, T., and Enami, I. (1997). The mechanism of the degradation of psaB gene product, one of the photosynthetic reaction center subunits of photosystem I, upon photoinhibition. Photosynth. Res. 53, 55-63. doi: 10.1023/A:1005852330671

Sonoike, K., and Terashima, I. (1994). Mechanism of photosystem-I photoinhibition in leaves of Cucumis sativus L. Planta 194, 287-293. doi: 10.1007/BF01101690

Suorsa, M., Jarvi, S., Grieco, M., Nurmi, M., Pietrzykowska, M., Rantala, M., et al. (2012). Proton gradient regulation5 is essential for proper acclimation of Arabidopsis photosystem I to naturally and artificially fluctuating light conditions. Plant Cell 24, 2934-2948. doi: 10.1105/tpc.112. 097162

Takahashi, S., Milward, S. E., Fan, D. Y., Chow, W. S., and Badger, M. R. (2009). How does cyclic electron flow alleviate photoinhibition in Arabidopsis? Plant Physiol. 149, 1560-1567. doi: 10.1104/pp.108.134122

Terashima, I., Funayama, S., and Sonoike, K. (1994). The site of photoinhibition in leaves of Cucumis sativus L. at low temperatures is photosystem I, not photosystem II. Planta 193, 300-306. doi: 10.1007/BF00192544 
Tikkanen, M., and Aro, E. M. (2014). Integrative regulatory network of plant thylakoid energy transduction. Trends Plant Sci. 19, 10-17. doi: 10.1016/j.tplants.2013.09.003

Tikkanen, M., Grieco, M., Kangasjarvi, S., and Aro, E. M. (2010). Thylakoid protein phosphorylation in higher plant chloroplasts optimizes electron transfer under fluctuating light. Plant Physiol. 152, 723-735. doi: 10.1104/pp.109. 150250

Tikkanen, M., Mekala, N. R., and Aro, E. M. (2014). Photosystem II photoinhibition-repair cycle protects photosystem I from irreversible damage. Biochim. Biophys. Acta 1837, 210-215. doi: 10.1016/j.bbabio.2013.10.001

Wang, P., Duan, W., Takabayashi, A., Endo, T., Shikanai, T., Ye, J. Y., et al. (2006). Chloroplastic NAD(P)H dehydrogenase in tobacco leaves functions in alleviation of oxidative damage caused by temperature stress. Plant Physiol. 141, 465-474. doi: 10.1104/pp.105.070490

Yamori, W., Evans, J. R., and von Caemmerer, S. (2010a). Effects of growth and measurement light intensities on temperature dependence of $\mathrm{CO}_{2}$ assimilation rate in tobacco leaves. Plant Cell Environ. 33, 332-343. doi: 10.1111/j.13653040.2009.02067.x

Yamori, W., Noguchi, K., Hikosaka, K., and Terashima, I. (2010b). Phenotypic plasticity in photosynthetic temperature acclimation among crop species with different cold tolerances. Plant Physiol. 152, 388-399. doi: 10.1104/pp.109.145862

Yamori, W., Sakata, N., Suzuki, Y., Shikanai, T., and Maniko, A. (2011). Cyclic electron flow around photosystem I via chloroplast $\mathrm{NAD}(\mathrm{P}) \mathrm{H}$ dehydrogenase $(\mathrm{NDH})$ complex performs a significant physiological role during photosynthesis and plant growth at low temperature in rice. Plant J. 68, 966-976. doi: 10.1111/j.1365-313X.2011.04747.x

Zhang, S. P., and Scheller, H. V. (2004). Photoinhibition of photosystem I at chilling temperature and subsequent recovery in Arabidopsis. Plant Cell Physiol. 45, 1595-1602. doi: $10.1093 / \mathrm{pcp} / \mathrm{pch} 180$
Zhang, W., Huang, W., Yang, Q. Y., Zhang, S. B., and Hu, H. (2013). Effect of growth temperature on the electron flow for photorespiration in leaves of tobacco grown in the field. Physiol. Plant. 149, 141-150. doi: 10.1111/ppl.12044

Zhang, Z., Jia, Y., Gao, H., Zhang, L., Li, H., and Meng, Q. (2011). Characterization of PSI recovery after chilling-induced photoinhibition in cucumber (Cucumis sativus L.) leaves. Planta 234, 883-889. doi: 10.1007/s00425-0111447-3

Zivcak, M., Brestic, M., Balatova, Z., Drevenakova, P., Olsovska, K., Kalaji, H. M., et al. (2013). Photosynthetic electron transport and specific photoprotective responses in wheat leaves under drought stress. Photosynth. Res. 117, 529-546. doi: 10.1007/s11120-013-9885-3

Zivcak, M., Brestic, M., Kalaji, H. M., and Govindjee. (2014b). Photosynthetic responses of sun- and shade-grown barley leaves to high light: is the lower PSII connectivity in shade leaves associated with protection against excess of light? Photosynth. Res. 119, 339-354. doi: 10.1007/s11120-014-9969-8

Zivcak, M., Kalaji, H. M., Shao, H.-B., Olsovska, K., and Brestic, M. (2014a). Photosynthetic proton and electron transport in wheat leaves under prolonged moderate drought stress. J. Photochem. Photobiol. B Biol. 137, 107-115. doi: 10.1016/j.jphotobiol.2014.01.007

Conflict of Interest Statement: The authors declare that the research was conducted in the absence of any commercial or financial relationships that could be construed as a potential conflict of interest.

Copyright (c) 2016 Huang, Yang, Hu and Zhang. This is an open-access article distributed under the terms of the Creative Commons Attribution License (CC BY). The use, distribution or reproduction in other forums is permitted, provided the original author(s) or licensor are credited and that the original publication in this journal is cited, in accordance with accepted academic practice. No use, distribution or reproduction is permitted which does not comply with these terms. 